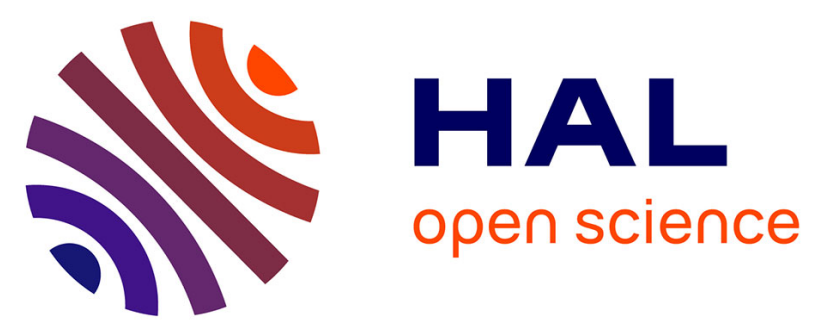

\title{
An algebraic approach for human posture estimation in the sagittal plane using accelerometer noisy signal
}

Wilfrid Perruquetti, Mamadou Mboup, Rosane Ushirobira, Philippe Fraisse, Vincent Bonnet

\section{- To cite this version:}

Wilfrid Perruquetti, Mamadou Mboup, Rosane Ushirobira, Philippe Fraisse, Vincent Bonnet. An algebraic approach for human posture estimation in the sagittal plane using accelerometer noisy signal. CDC: Conference on Decision and Control, Dec 2012, Maui, HI, United States. pp.7389-7394, 10.1109/CDC.2012.6426001 . hal-00733516

\section{HAL Id: hal-00733516 https://hal.inria.fr/hal-00733516}

Submitted on 18 Sep 2012

HAL is a multi-disciplinary open access archive for the deposit and dissemination of scientific research documents, whether they are published or not. The documents may come from teaching and research institutions in France or abroad, or from public or private research centers.
L'archive ouverte pluridisciplinaire HAL, est destinée au dépôt et à la diffusion de documents scientifiques de niveau recherche, publiés ou non, émanant des établissements d'enseignement et de recherche français ou étrangers, des laboratoires publics ou privés. 


\title{
An algebraic approach for human posture estimation in the sagittal plane using accelerometer noisy signal*
}

\author{
Wilfrid Perruquetti ${ }^{1}$, Vincent Bonnet ${ }^{2}$, Mamadou Mboup $^{3}$, Rosane Ushirobira ${ }^{4}$ and Philippe Fraisse ${ }^{5}$
}

\begin{abstract}
Our aim is to develop an algebraic approach to estimate human posture in the sagittal plane using Inertial Measurement Unit (IMU) providing accelerations and angular velocities. For this purpose, we address the issue of the estimation of the amplitude, frequency and phase of a biased and noisy sum of three sinusoidal waveform signals on a moving time horizon. The length of the time window being small, the estimation must be done within a fraction of the signal's period. The problem is solved via an algebraic method. The efficiency of our approach is illustrated by computer simulations.
\end{abstract}

\section{INTRODUCTION}

In human motion analysis, kinematics and kinetics quantities are usually obtained by using a stereophotogrammetric system and force-plates providing accurate results but requiring a considerable economic investment and complex experimental protocols. Inertial measurement units (IMUs) have recently became popular as an instrument to quantify human motion [7], thanks to their ease-of-use, robust design, low-cost and small dimensions. Additionally, mass market electronics such as cell phones contain embedded IMUs along with recording and transmission capabilities. For these reasons, IMUs are not confined to the laboratory, opening a wide field of possibilities in rehabilitation.

Usually, IMUs include accelerometers and rate gyroscopes to measure accelerations and angular velocities, respectively. Theoretically, the determination of the position and orientation of the device in space could be obtained by integrating the obtained signals. Unfortunately, the outputs are subject to drift over time and that jeopardizes the time integration of the raw signals when estimating orientation data [7]. This problem has been overcome by the use of recursive filters, such as Kalman filters [7], allowing the estimation of the sensor orientation only, if no external sensor is used, i.e. a magnetometer. Model based adaptive filters, i.e. Fourier Linear Combiner [31], were used to estimate a trunk orientation during walking [4], but appeared to be sensitive

\footnotetext{
*This work was supported by Inria Lille - Nord Europe.

1 Wilfrid Perruquetti is with Université Lille Nord de France \& École Centrale de Lille \& Non-A Inria Lille - Nord Europe \& LAGIS (CNRS), France (e-mail: Wilfrid.Perruquettieinria.fr).

2 Vincent Bonnet is with LABLAB \& Department of Human Movement and Sports Sciences \& University of Rome Foro Italico, Italy. (e-mail: v.bonneteuniroma 4.it).

3 Mamadou Mboup is with CReSTIC - Université de Reims Champagne Ardenne \& Non-A Inria Lille - Nord Europe, France (e-mail: Mamadou. Mboup@univ-reims. fr).

4 Rosane Ushirobira is with Institut de Mathématiques de Bourgogne (CNRS) - Université de Bourgogne \& Non-A Inria Lille - Nord Europe, France (e-mail: Rosane.Ushirobira@inria.fr).

5 Philippe Fraisse is with LIRMM (CNRS) \& Universit Montpellier 2, France (e-mail: fraisseelirmm.fr).
}

to parameter choices and conditions of use, thus highly task constrained.

Movement analysis literature provides numerous examples where several IMUs are attached to adjacent body segments to obtain 3D joint kinematics [22], [18]. For clinical or sports applications in a daily environment, and in the framework of a minimum measured input model proposed by Cappozzo [6] to maximize the functional information extracted from simplified experimental protocols, it is desirable to reduce the number of devices to a minimum.

Recently, accurate methodology [5] has been proposed to estimate kinematics, i.e. ankle, knee and hip angles, and kinetics in the sagittal plane, by using only one IMU located at the lower trunk. This approach, based on the leastsquare minimization of the difference between measured and estimated linear accelerations and the angular velocity at the IMU site, was validated through a body weight squat exercise stating the pseudo-periodicity of this task. However, this numerical approach was computationally time demanding and can be used only a posteriori of the actual motion performed by the human.

The squat exercise is one of the most popular movement used in rehabilitation [1] since it involves multiple joints and muscles in a single motion [17] and it is hence considered as very effective for improving lower-limb muscle function. Furthermore, the ability to perform this task is a prerequisite for more complex skills of daily living such as picking up an item, descending stairs, or rising from a chair [1]. As a consequence, the squat exercise is included in rehabilitation protocols [1], sports training programs [9] and assessment protocols for pathologies [2], [8].

This paper investigates the possibility of estimating lowerlimb kinematics in the sagittal plane during a squat exercise using data collected by a single IMU, located on the lower trunk, and a three-degrees-of-freedom model of the musculoskeletal system. Remark that the IMU provides three independent kinematic quantities within a plane (an angular velocity and two acceleration components).

This issue is formulated in this paper as a parameter estimation problem of a sum of three complex exponential sinusoidal signals. This famous estimation problem was notably studied by Riche de Prony in his 1795 seminal paper [30] (see [21], [28] for more recent works). Several other resolution methods have been elaborated, such as linear or nonlinear regression, subspace methods [19], [32], [21], the extended Kalman filter [3], the notches filter [29], or techniques from adaptive nonlinear control [20], [26]. Nevertheless, the parameter estimation problem in a fraction 
of the time signal, from a biased and noisy signal has not been entirely solved. Our solution draws its inspiration from the algebraic analysis of [14], [13], [15], [11], [24]. More encouraging results in concrete examples can be found in [27], [33], [34], [36].

Section II goes from the geometric model of the body in the sagittal plane to the parameter estimation problem. Section III provides the estimation of the desired parameters. Section IV contains convincing numerical experiments that illustrate our method and compare it to the well-known modified Prony's method.

\section{PROBLEM FORMULATION}

As we mentioned earlier, the squat exercise is one of the most popular movement used in rehabilitation. In this setting, we would like to estimate kinematics, i.e. knee and hip angles in the sagittal plane using a single IMU located at the lower trunk. For this, let us consider a simplified 2D geometric model of a 3-segmented body (such a simplified model is currently used in biomechanics studies). This model is described by the figure below where the joint positions are:

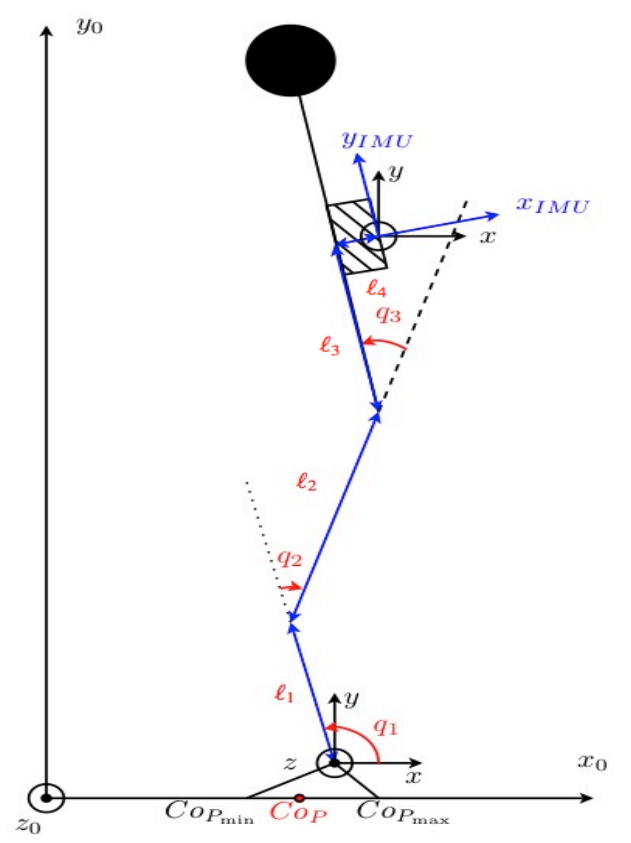

Fig. 1. Geometric model in the sagittal plane

$q=\left(q_{1}, q_{2}, q_{3}\right)^{T}$. Assume that $\ell_{1}, \ell_{2}$ and $\ell_{3}$ are unknown. Our geometric model is given by:

$$
\begin{aligned}
& x=\ell_{1} \mathrm{c}_{1}+\ell_{2} \mathrm{c}_{2}+\ell_{3} \mathrm{c}_{3}+\ell_{4} \mathrm{~s}_{3} \\
& y=\ell_{1} \mathrm{~s}_{1}+\ell_{2} \mathrm{~s}_{2}+\ell_{3} \mathrm{~s}_{3}-\ell_{4} \mathrm{c}_{3}
\end{aligned}
$$

where $\mathrm{c}_{k}=\sum_{j=1}^{k} \cos \left(q_{j}\right)$ and $\mathrm{s}_{k}=\sum_{j=1}^{k} \sin \left(q_{j}\right)$, for $k=$ 1, 2, 3. Some hypothesis are enforced for such a movement: on a moving small time window, each angle is approximated by a line. That is:

$$
\begin{aligned}
q_{1}(u) & =\gamma_{1}(u)=\omega_{1} u+\phi_{1}, \\
\left(q_{1}+q_{2}\right)(u) & =\gamma_{2}(u)=\omega_{2} u+\phi_{2}, \\
\left(q_{1}+q_{2}+q_{3}\right)(u) & =\gamma_{3}(u)=\omega_{3} u+\phi_{3}
\end{aligned}
$$

for $u \in[t-T, t]$ where $t$ is the current time and $T$ is the time window length. Using the complex notation:

$$
\begin{aligned}
p(t)= & x(t)+i y(t) \\
= & \ell_{1} \exp \left(i \gamma_{1}\right)+\ell_{2} \exp \left(i \gamma_{2}\right)+\left(\ell_{3}-i \ell_{4}\right) \exp \left(i \gamma_{3}\right) \\
= & \ell_{1} \exp \left(i\left(\omega_{1} t+\phi_{1}\right)\right)+\ell_{2} \exp \left(i\left(\omega_{2} t+\phi_{2}\right)\right) \\
& \quad+\left(\ell_{3}-i \ell_{4}\right) \exp \left(i\left(\omega_{3} t+\phi_{3}\right)\right) .
\end{aligned}
$$

Taking the time derivative of (1), one obtains:

$$
\begin{aligned}
\frac{d^{n+2} p}{d t^{n+2}}= & \left(i \omega_{1}\right)^{n+2} \ell_{1} \exp \left(i \gamma_{1}\right)+\left(i \omega_{2}\right)^{n+2} \ell_{2} \exp \left(i \gamma_{2}\right) \\
& +\left(i \omega_{3}\right)^{n+2}\left(\ell_{3}-i \ell_{4}\right) \exp \left(i \gamma_{3}\right)
\end{aligned}
$$

for $n \in \mathbb{N}$. Using the measured acceleration $m_{a}=\ddot{p}+\varpi$ where $\varpi$ is the noise and $p$ is the position in the sagittal plane written as $p=x+i y$ (see (1)), we would like to obtain an estimation of the parameters involved in the joint position description.

Replace $n$ by 0,1 and 2 in (2) to check that the acceleration $a_{c c}=\ddot{p}$ satisfies:

$$
a_{c c}^{(3)}+\theta_{3} a_{c c}^{(2)}+\theta_{2} a_{c c}^{(1)}+\theta_{1} a_{c c}=0
$$

with

$$
\begin{aligned}
& \theta_{1}=i \omega_{1} \omega_{2} \omega_{3}, \\
& \theta_{2}=-\left(\omega_{1} \omega_{2}+\omega_{1} \omega_{3}+\omega_{2} \omega_{3}\right), \\
& \theta_{3}=-i\left(\omega_{1}+\omega_{2}+\omega_{3}\right) .
\end{aligned}
$$

\section{SOLUTION WITHIN AN ALGEBRAIC FRAMEWORK}

Our aim is to reconstruct the position by estimating its parameters on a moving time horizon. Since the position is:

$$
p(t)=\sum_{k=1}^{3} \alpha_{k} \exp \left(i\left(\omega_{k} t+\phi_{k}\right)\right)
$$

where the parameters are given by $\alpha_{1}=\ell_{1}, \alpha_{2}=$ $\ell_{2}, \alpha_{3}=\left(\ell_{3}-i \ell_{4}\right)$, we want to estimate the parameters $\left(\alpha_{k}, \omega_{k}, \phi_{k}\right)_{k=1 \ldots 3}$ from the biased and noisy output measure

$$
y(t)=a_{c c}(t)+\beta+\varpi,
$$

where $a_{c c}=\ddot{p}$ (the only measured variable is the acceleration), $\beta$ is an unknown constant bias and $\varpi$ is a noise $^{1}$. A linear parametric estimation problem may often be formalized as finding a good approximation of some vector $\Theta$ on the basis of an observed signal which is a linear functional of the "true" signal depending on a set of parameters and a noise corrupting the observation. Here the signal $z(t)=a_{c c}(t)+\beta$ and $\Theta$ satisfy the differential equation (see 3$)$ :

$$
z^{(3)}(t)+\theta_{3} z^{(2)}(t)+\theta_{2} \dot{z}(t)+\theta_{1}(z(t)-\beta)=0 .
$$

where are given by (4)-(6). This differential equation, in the operational domain, reads as:

\footnotetext{
${ }^{1}$ We use here the framework developed in [10], [11], independent of any probabilistic modeling of the noise. In this point of view, the noise is seen as a fast oscillation (see [10]).
} 


$$
\begin{aligned}
& s\left(s-i \omega_{1}\right)\left(s-i \omega_{2}\right)\left(s-i \omega_{3}\right) Z(s)= \\
& s\left(s^{2}-i\left(\omega_{1}+\omega_{2}+\omega_{3}\right) s-\left(\omega_{1} \omega_{2}+\omega_{1} \omega_{3}+\omega_{2} \omega_{3}\right)\right) z(0) \\
& s\left(s+i \omega_{1} \omega_{2} \omega_{3}\right) \dot{z}(0)+s \ddot{z}(0)-\beta \omega_{1} \omega_{2} \omega_{3}
\end{aligned}
$$

Among the unknown parameters, we wish to estimate $\Theta_{\mathrm{est}}:=\left\{\theta_{1}, \theta_{2}, \theta_{3}, \theta_{4}, \theta_{5}, \theta_{6}\right\}$, but not the bias $\Theta_{\overline{\mathrm{est}}}=\left\{\theta_{7}\right\}$, where

$$
\begin{aligned}
\theta_{4} & =-a_{c c}(0)=\beta-z(0), \\
\theta_{5} & =-\dot{z}(0)=-\dot{a}_{c c}(0), \\
\theta_{6} & =-\ddot{z}(0)=-\ddot{a}_{c c}(0), \\
\theta_{7} & =-\beta
\end{aligned}
$$

Notice that the original parameters can be easily deduced from $\Theta_{\text {est }}$.

Lemma 1: The parameters $\ell_{k}, \omega_{k}, \phi_{k}, k=1 \ldots 3$ and $\ell_{4}$ can be obtained from $\Theta_{\text {est }}$.

Proof: Let us note that $\ell_{1}=\alpha_{1}, \ell_{2}=\alpha_{2}, \ell_{3}=\Re\left(\alpha_{3}\right)$, $\ell_{4}=-\Im\left(\alpha_{3}\right)$. If $k=1,2,3$, one can easily deduce $\omega_{k}$ from $\theta_{k}$ since $i \omega_{k}$ are the roots of the polynomial $\omega^{3}+$ $\theta_{3} \omega^{2}+\theta_{2} \omega+\theta_{1}=0$. From $\theta_{4}, \theta_{5}$ and $\theta_{6}$, one obtains $a_{c c}(0)=-\theta_{4}=\sum_{k=1}^{3}\left(i \omega_{k}\right)^{2} \alpha_{k} \exp i \phi_{k}, \dot{a}_{c c}(0)=-\theta_{5}=$ $\sum_{k=1}^{3}\left(i \omega_{k}\right)^{3} \alpha_{k} \exp i \phi_{k}$ and ${ }_{6}=\sum_{k=1}^{3}\left(i \omega_{k}\right)^{3} \alpha_{k} \exp i \phi_{k}$ :

$$
\left(\begin{array}{c}
-\theta_{4} \\
\theta_{5} \\
\theta_{5}
\end{array}\right)=A\left(\begin{array}{c}
\alpha_{1} \exp i \phi_{1} \\
\alpha_{2} \exp i \phi_{2} \\
\alpha_{3} \exp i \phi_{4}
\end{array}\right)
$$

with $A=\left(\begin{array}{lll}\left(i \omega_{1}\right)^{2} & \left(i \omega_{2}\right)^{2} & \left(i \omega_{3}\right)^{2} \\ \left(i \omega_{1}\right)^{3} & \left(i \omega_{2}\right)^{3} & \left(i \omega_{2}\right)^{3} \\ \left(i \omega_{1}\right)^{4} & \left(i \omega_{2}\right)^{4} & \left(i \omega_{2}\right)^{3}\end{array}\right)$ a Vandermonde matrix. Taking its inverse gives $\alpha_{k} \exp i \phi_{k}, k=1 \ldots 3$. The desired results are obtained by taking absolute values and arguments of these complex relations and by ordering the frequencies.

Using the notation (4)-(8), the equation (7) reads as

$$
\begin{aligned}
& s\left(s^{3}+\theta_{3} s^{2}+\theta_{2} s+\theta_{1}\right) Z(s)+s\left(s^{2}+\theta_{3} s+\theta_{2}\right) \theta_{4} \\
& s\left(s+\theta_{3}\right) \theta_{5}+s \theta_{6}+\left(s^{3}+\theta_{3} s^{2}+\theta_{2} s+\theta_{1}\right) \theta_{7}=0,
\end{aligned}
$$

from which we would like to obtain a system of equations on $\Theta_{\text {est }}$ and independent of $\Theta_{\overline{\text { est }}}$. We denote by $\mathbb{C}_{\Theta}[s]^{2}$ (respectively $\mathbb{C}_{\Theta_{\text {est }}}[s]$ ) the polynomial ring in the variable $s$ with coefficients in $\mathbb{C}_{\Theta}$ (respectively in $\mathbb{C}_{\Theta_{\text {est }}}$ ) where $\mathbb{C}_{\Theta}:=\mathbb{C}(\Theta)$ and $\mathbb{C}_{\Theta_{\text {est }}}:=\mathbb{C}\left(\Theta_{\text {est }}\right)$ are algebraic extensions. We obtain the relation:

$$
\mathcal{R}\left(s, Z(s), \Theta_{\text {est }}, \Theta_{\overline{\text { est }}}\right):=P(s) Z(s)+Q(s)+\bar{Q}(s)=0
$$

with

${ }^{2}$ The polynomial ring in $\frac{d}{d s}$ with coefficients in $\mathbb{C}_{\Theta}[s]$.

$$
\begin{aligned}
& P(s)=s T(s) \\
& Q(s)=s^{3} \theta_{4}+s^{2}\left(\theta_{5}+\theta_{3} \theta_{4}\right)+s\left(\theta_{6}+\theta_{2} \theta_{4}+\theta_{3} \theta_{5}\right) \\
& \bar{Q}(s)=T(s) \theta_{7} \in \mathbb{C}_{\Theta}[s] \quad
\end{aligned}
$$

where we set $T(s)=s^{3}+\theta_{3} s^{2}+\theta_{2} s+\theta_{1} \in \mathbb{C}_{\Theta_{\text {est }}}[s]$. We proceed in three steps:

1) Algebraic elimination of all terms in $\Theta_{\overline{\mathrm{est}}}$ : let us notice that when $\bar{Q}$ is a constant, the differential operator $\frac{d}{d s}$ annihilates it, since $\frac{d}{d s} \bar{Q}=0$. More generally, there exist differential operators that annihilate any $\bar{Q}$ (called $\bar{Q}$-annihilators). All possible $\bar{Q}$-annihilators form a principal ideal of $\mathbb{C}_{\Theta_{\mathrm{est}}}(s)\left[\frac{d}{d s}\right]$ (meaning this set is generated by a single operator in $\mathbb{C}_{\Theta_{\text {est }}}(s)\left[\frac{d}{d s}\right]$ ). This generator is called a $\bar{Q}$-minimal annihilator.

2) Obtaining a system of equations in $\Theta_{\text {est }}$ : a minimal annihilator generates all differential operators that annihilate $\bar{Q}$. Exploiting the Weyl Algebra structure of $\mathbb{C}_{\Theta_{\text {est }}}(s)\left[\frac{d}{d s}\right]$, any $\bar{Q}$ - annihilator is rewritten in a canonical form. That allows us to make choices in order to obtain a system of equations having good numerical properties (once back in the time domain).

3) Resolution of the obtained system: we use the inverse Laplace transform

$\mathcal{L}^{-1}\left(\frac{1}{s^{m}} \frac{d^{p} Z(s)}{d s^{p}}\right)=\frac{(-1)^{p} t^{m+p}}{(m-1) !} \int_{0}^{1} w_{m-1, p}(\tau) z(t \tau) d \tau$

with $w_{m, p}(t)=(1-t)^{m} t^{p}, \forall p, m \in \mathbb{N}, m \geq 1$ to bring the equations back in the time domain. The integers $m, p$ will be chosen as small as possible so that the resulting estimation is as least as possible sensitive to the noise.

To briefly explain the theoretical framework, let us set $\mathrm{B}:=\mathbb{C}(s)\left[\frac{d}{d s}\right]$. This algebra is endowed with a structure of a one-generator Weyl algebra. In consequence, it benefits of nice properties of this algebraic structure, such as the principality of left ideals.

Definition 2: Let $R \in \mathbb{C}_{\Theta}[s]$. An $R$-annihilator w.r.t. $\mathrm{B}$ is an element of $\operatorname{Ann}_{\mathrm{B}}(R)=\{F \in \mathrm{B} \mid F(R)=0\}$.

Since $\operatorname{Ann}_{\mathrm{B}}(R)$ is a left ideal, then $\operatorname{Ann}_{\mathrm{B}}(R)$ is a principal left ideal, i.e. it is generated by a unique $\Pi_{\min } \in \mathrm{B}$, up to multiplication by an element of $\mathrm{B}$. That means

$$
\operatorname{Ann}_{\mathrm{B}}(R)=\mathrm{B} \Pi_{\min } \text {. }
$$

We call $\Pi_{\min }$ a minimal $R$-annihilator w.r.t. $\mathrm{B}$. Remark that $\operatorname{Ann}_{\mathrm{B}}(R)$ contains annihilators in finite integral form, i.e. operators with coefficients in $\mathbb{C}\left[\frac{1}{s}\right]$. We have the following lemmas:

Lemma 3: Let $R=s^{n}, n \in \mathbb{N}$. A minimal $R$-annihilator is given by

$$
\Pi_{n}=s \frac{d}{d s}-n
$$


Moreover, for $m, n \in \mathbb{N}$, the operators $\Pi_{m}$ and $\Pi_{n}$ commute. Thus, one can use the following Lemma

Lemma 4: Let $P_{1}$ and $P_{2} \in \mathbb{C}_{\Theta}[s]$. Let $\Pi_{1}$ be a $P_{1^{-}}$ annihilator and $\Pi_{2}$ be a $P_{2}$-annihilator such that $\Pi_{1} \Pi_{2}=$ $\Pi_{2} \Pi_{1}$. Then $\Pi_{1} \Pi_{2}$ is a $\left(\mu P_{1}+\eta P_{2}\right)$-annihilator for all $\mu, \eta \in \mathbb{C}_{\Theta}$.

Now, recall that $\bar{Q}=\left(s^{3}+\theta_{3} s^{2}+\theta_{2} s+\theta_{1}\right) \theta_{7}$. So, the above Lemma provides a minimal $\bar{Q}$-annihilator w.r.t. B:

$$
\Pi_{\min }=\left(s \frac{d}{d s}-4\right) \circ \cdots \circ\left(s \frac{d}{d s}-1\right) \circ\left(s \frac{d}{d s}\right) .
$$

Using the commuting rules in $\mathrm{B}$, it is not hard to prove that:

$$
\Pi_{\min }=s^{4} \frac{d^{4}}{d s^{4}} .
$$

Applying $\Pi_{\min }$ on relation (8) gives

$$
\begin{aligned}
& \Pi_{\min }(P(s) Z(s))=P_{4}(s) \frac{d^{4} Z(s)}{d s^{4}}+P_{3}(s) \frac{d^{3} Z(s)}{d s^{3}} \\
& +P_{2}(s) \frac{d^{2} Z(s)}{d s^{2}}+P_{1}(s) \frac{d Z(s)}{d s}+P_{0}(s) Z(s)
\end{aligned}
$$

where

$$
\begin{aligned}
& P_{4}(s)=s^{5} T(s), \\
& P_{3}(s)=s^{5}\left(16 s^{2}+12 s \theta_{3}+\theta_{2}\right), \\
& P_{2}(s)=s^{4}\left(72 s^{2}+36 s \theta_{3}+12 \theta_{2}\right), \\
& P_{1}(s)=s^{4}\left(96 s+24 \theta_{3}\right), \\
& P_{0}(s)=24 s^{4}, \\
& \Pi_{\text {min }}(Q(s))=0, \quad \Pi_{\text {min }}(\bar{Q}(s))=0 .
\end{aligned}
$$

providing the following algebraic relation

$$
\sum_{k=0}^{4} P_{k}(s) \frac{d^{k} Z(s)}{d s^{k}}=0 .
$$

We obtain a single equation in $\theta_{1}, \theta_{2}$ and $\theta_{3}$. To linearly identify these three parameters, we need two independent equations. The following result show that this is not possible in the operational domain.

Theorem 5: There do not exist three $\bar{Q}$-annihilators w.r.t $\mathbb{C}(s)\left[\frac{d}{d s}\right]$ leading to three independent equations in $\theta_{1}, \theta_{2}$ and $\theta_{3}$.

Proof: Thanks to the principality of the left ideal $\operatorname{Ann}_{\mathrm{B}}(\bar{Q})$, any $\bar{Q}$-annihilator is of the form

$$
\Pi=\sum_{i=0}^{\ell} g_{i}(s) \frac{d^{i+4}}{d s^{i+4}}
$$

where $g_{i}(s) \in \mathbb{C}_{\Theta_{\text {est }}}(s), \forall 1 \leq i \leq \ell$. We have $\Pi(Q(s))=$ $\Pi(\bar{Q})=0$. Since $P \in \mathbb{C}[s]$ is of degree 4 , by computing $\Pi(P(s) Z(s))$ we obtain:

$$
\frac{d^{i+4}}{d s^{i+4}}(P(s) Z(s))=\sum_{k=0}^{4}\left(\begin{array}{c}
i+4 \\
k
\end{array}\right) P^{(k)}(s) Z^{(i+4-k)}(s)
$$

with

$$
\begin{aligned}
& P^{(0)}(s)=s^{4}+\theta_{3} s^{3}+\theta_{2} s^{2}+\theta_{1} s \\
& P^{(1)}(s)=4 s^{3}+3 \theta_{3} s^{2}+2 \theta_{2} s+\theta_{1} \\
& P^{(2)}(s)=12 s^{2}+6 \theta_{3} s+2 \theta_{2} \\
& P^{(3)}(s)=24 s+6 \theta_{3} \\
& P^{(4)}(s)=24
\end{aligned}
$$

Therefore $\Pi$ applied on relation $\mathcal{R}(8)$ reads as:

$$
\sum_{i=0}^{\ell} g_{i}(s)\left(A_{i} \theta_{1}+B_{i} \theta_{2}+C_{i} \theta_{3}+D_{i}\right)=0
$$

where $A_{i}, B_{i}, C_{i}$ and $D_{i}$ depend on the variables $s$ and $\frac{d}{d s}$. Denote by $L_{i}:=\left(A_{i} B_{i} C_{i} D_{i}\right)$. Then for $i>j$ we have by induction on $(j-i)$ :

$$
L_{j}=\frac{d}{d s}^{j-i} L_{i}
$$

and that completes the proof of the theorem.

Remark 6: Let us note that for a similar parameter identification problem of a sinusoid, it is possible to find two independent equations in the operational domain (see [37]).

Therefore we are going to use such a construction in the time domain. To obtain three equations we select $\ell=2$ in the expression of a $\bar{Q}$-annihilator. That leads to the following $6^{\text {th }}$-order annihilator written in the canonical form:

$$
\Pi=g_{0}(s) \frac{d^{4}}{d s^{4}}+g_{1}(s) \frac{d^{5}}{d s^{5}}+g_{2}(s) \frac{d^{6}}{d s^{6}},
$$

where $g_{0}(s), g_{1}(s), g_{2}(s) \in \mathbb{C}(s)$. The choice of $g_{0}(s)=$ $1, g_{1}(s)=0, g_{2}(s) ; g_{0}(s)=0, g_{1}(s)=1, g_{2}(s)=0$ and $g_{0}(s)=0, g_{1}(s)=0, g_{2}(s)=1$ gives three equations in the operational domain leading to the following system in the time domain:

$$
\left(\begin{array}{ccc}
\frac{1}{6} J_{1} & \frac{1}{6} J_{2} & J_{3} \\
-\frac{1}{24} J_{4} & -\frac{1}{6} J_{5} & -\frac{1}{2} J_{6} \\
\frac{1}{12} J_{7} & \frac{1}{4} J_{8} & J_{9}
\end{array}\right)\left(\begin{array}{l}
\theta_{1} \\
\theta_{2} \\
\theta_{3}
\end{array}\right)=-\left(\begin{array}{l}
J_{10} \\
J_{11} \\
J_{12}
\end{array}\right)
$$

where we set $v_{m, n}=v_{m, n}(u)=(t-u)^{m} u^{n}$, for $m, n \in \mathbb{N}$ and $J_{i}=\int_{0}^{t} I_{i} z(u) d u$, for $1 \leq i \leq 12$, 


$$
\begin{aligned}
I_{1} & =2 v_{3,4}-t v_{3,3} \\
I_{2} & =14 v_{2,4}-14 t v_{2,3}+3 t^{2} v_{2,2} \\
I_{3} & =14 v_{1,4}-21 t v_{1,3}+9 t^{2} v_{1,2}-t^{3} v_{1,1} \\
I_{4} & =9 v_{3,5}-5 t v_{3,4} \\
I_{5} & =18 v_{2,5}-20 t v_{2,4}+5 t^{2} v_{2,3} \\
I_{6} & =42 v_{1,5}-70 t v_{1,4}+35 t^{2} v_{1,3}-5 t^{3} v_{1,2} \\
I_{7} & =5 v_{3,6}-3 t v_{3,5} \\
I_{8} & =15 v_{2,6}-18 t v_{2,5}+5 t^{2} v_{2,4} \\
I_{9} & =30 v_{1,6}-54 t v_{1,5}+30 t^{2} v_{1,4}-5 t^{3} v_{1,3} \\
I_{10} & =-16 v_{1,3}+36 v_{2,2}-16 v_{3,1}+v_{0,4}+v_{4,0} \\
I_{11} & =20 v_{1,4}-60 v_{2,3}+40 v_{3,2}-5 v_{4,1}-v_{0,5} \\
I_{12} & =-24 v_{1,5}+15 v_{4,2}+90 v_{2,4}-80 v_{3,3}+v_{0,6}
\end{aligned}
$$

Finally, the expressions for $\theta_{1}, \theta_{2}$ and $\theta_{3}$ are:

$$
\begin{gathered}
\theta_{1}=12 \frac{2 a J_{2}-2 b J_{3}+d J_{10}}{\Lambda} \\
\theta_{2}=12 \frac{-2 a J_{1}+c J_{3}+e J_{10}}{\Lambda} \\
\theta_{3}=\frac{4 b J_{1}-2 c J_{2}+f J_{10}}{\Lambda}
\end{gathered}
$$

where

$$
\begin{aligned}
& a=2 J_{9} J_{11}+J_{6} J_{12}, b=3 J_{8} J_{11}+2 J_{5} J_{12}, \\
& c=2 J_{7} J_{11}+J_{4} J_{12}, d=4 J_{5} J_{9}-3 J_{6} J_{8}, \\
& e=J_{6} J_{7}-J_{4} J_{9}, f=3 J_{4} J_{8}-4 J_{5} J_{7}, \\
& \Lambda=2 d J_{1}+2 e J_{2}+f J_{3}
\end{aligned}
$$

\section{A. Estimation of $\theta_{4}, \theta_{5}$ and $\theta_{6}$}

Using annihilators generated by the minimal $\bar{Q}$-annihilator w.r.t. $\mathbb{C}(s)\left[\frac{d}{d s}\right]$, we could linearly identify the parameters $\theta_{1}, \theta_{2}$ and $\theta_{3}$. These annihilators do not depend on the parameters to be found. Now, one can show that it is not possible to identify linearly the remaining parameters $\theta_{4}, \theta_{5}$ and $\theta_{6}$, so we will use nonlinear equations in $\theta_{1}, \theta_{2}$ and $\theta_{3}$. Recall that $T(s)=s^{3}+\theta_{3} s^{2}+\theta_{2} s+\theta_{1} \in \mathbb{C}_{\Theta_{\text {est }}}[s]$. So, let us consider

$$
\Pi_{\mathrm{min}}^{\Theta_{\mathrm{est}}}=T \frac{d}{d s}-T^{\prime}
$$

a minimal $\bar{Q}$-annihilator w.r.t. $\mathbb{C}_{\Theta_{\text {est }}}(s)\left[\frac{d}{d s}\right]$. Applying it on relation (8) gives:

$$
\begin{aligned}
& \Pi_{\min }^{\Theta_{\text {est }}}(P Z)=T^{2}\left(s \frac{d Z}{d s}+Z\right) \\
& \Pi_{\min }^{\Theta_{\text {est }}}(Q)=T^{\prime} \theta_{1} \theta_{4}+T\left(U^{\prime}-U\right) \theta_{5}+T(1-s) \theta_{6} \\
& \Pi_{\min }^{\Theta_{\text {est }}}(\bar{Q})=0
\end{aligned}
$$

where $U=s^{2}+\theta_{3} s \in \mathbb{C}_{\Theta}[s]$. That gives the following algebraic relation:

$T^{2}\left(s \frac{d Z}{d s}+Z\right)+T^{\prime} \theta_{1} \theta_{4}+T\left(U^{\prime}-U\right) \theta_{5}+T(1-s) \theta_{6}=0$
We obtain a single equation in $\theta_{4}, \theta_{5}$ and $\theta_{6}$. That leads to the following annihilator:

$$
\begin{gathered}
\Pi=g_{0}(s) \Pi_{\mathrm{min}}^{\Theta_{\mathrm{est}}}+g_{1}(s)\left(T \frac{d^{2}}{d s^{2}}-T^{\prime \prime}\right)+ \\
g_{2}(s)\left(T \frac{d^{3}}{d s^{3}}+T^{\prime} \frac{d^{2}}{d s^{2}}-T^{\prime \prime} \frac{d}{d s}-T^{\prime \prime \prime}\right),
\end{gathered}
$$

where $g_{0}(s), g_{1}(s)$ and $g_{2}(s) \in \mathbb{C}(s)$. Selecting $g_{0}(s)=1$, $g_{1}(s)=0, g_{2}(s) ; g_{0}(s)=0, g_{1}(s)=1, g_{2}(s)=0$ and $g_{0}(s)=0, g_{1}(s)=0, g_{2}(s)=1$, we obtain three equations:

$$
\left(\begin{array}{ccc}
T^{\prime} \theta_{1} & T\left(U^{\prime}-U\right) & T(1-s) \\
T^{\prime \prime} \theta_{1} & 2 T-T^{\prime \prime} U & -s T^{\prime \prime} \\
6 \theta_{1} & 2 T^{\prime}-T^{\prime \prime} U^{\prime}-6 U & -T^{\prime \prime}-6 s
\end{array}\right)\left(\begin{array}{c}
\theta_{4} \\
\theta_{5} \\
\theta_{6}
\end{array}\right)=-\left(\begin{array}{c}
B_{1} \\
B_{2} \\
B_{3}
\end{array}\right)
$$

where

$$
\begin{aligned}
B_{1}= & T^{2}\left(s \frac{d Z}{d s}+Z\right) \\
B_{2}= & T\left(2 T^{\prime} Z+2\left(T+s T^{\prime}\right) \frac{d Z}{d s}+s T \frac{d^{2} Z}{d s^{2}}\right) \\
B_{3}= & 2\left(T T^{\prime \prime}+T^{\prime 2}\right) Z+2\left(s T T^{\prime \prime}+4 T T^{\prime}+s T^{\prime 2}\right) \frac{d Z}{d s} \\
& +T\left(3 T+4 s T^{\prime}\right) \frac{d^{2} Z}{d s^{2}}+s T^{2} \frac{d^{3} Z}{d s^{3}}
\end{aligned}
$$

After some elementary operations on the above matrix, a triangular matrix can be obtained providing the formulas for the remaining $\theta_{4}, \theta_{5}$ and $\theta_{6}$. These expressions are rather extensive, so we do not present them here.

\section{Simulations}

The following figures show the estimation of the parameters $\theta_{1}, \theta_{2}$ and $\theta_{3}$ concerning the results of the normalized mean values and variances. More precisely, the "true" parameters are denoted by $\theta_{1}, \theta_{2}$ and $\theta_{3}$ and $\widehat{\theta}_{i, k}$ denotes the estimation of $\theta_{i}$ obtained at the $k$-th run. The modified Prony's method (PM) is used as a reference. Each point is obtained by averaging the results over 100 trials.

Dotted line curves represent exact values, while solid line curves show the estimations by our algebraic method and dashed line curves, the results by the modified Prony's method.

The simulation results for the estimation of the parameters $\theta_{1}, \theta_{2}$ and $\theta_{3}$ versus the estimation time is shown in figure 2.

Figure 3 shows the plot of $\widehat{\theta}_{i}=\frac{1}{100} \sum_{k} \widehat{\theta}_{i, k}$ and $\frac{\operatorname{var}\left(\widehat{\theta}_{i}\right)}{\theta_{i}^{2}}$ versus the estimation time.

\section{REFERENCES}

[1] F. S. M. Alves, F. S. Oliveira, C. H. B. F. Junqueira, B. M. S. Azevedo and V. C. Dionísio Analysis of electromyographic patterns during standard and declined squats, Revista Brasileira Fisioterapia, vol. 13, pp. 164-172, 2009.

[2] D. R. Bell, D. A. Padua and M. A. Clark Muscle strength and flexibility characteristics of people displaying excessive medial knee displacement, Archive Physical Medicine and Rehabilitation, vol. 89, pp. 1323-1328, 2008.

[3] S. Bittanti and S. Savaresi, On the parameterization and design of an extended Kalman filter frequency tracker, IEEE Trans. Automat. Control, vol. 45, pp. 1718-1724, 2000. 

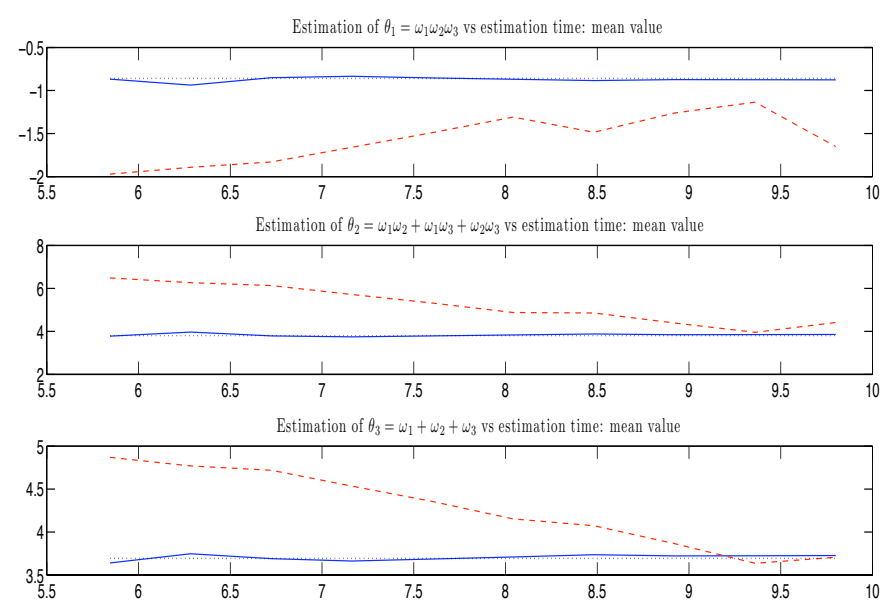

Fig. 2. Comparison with the modified Prony's method
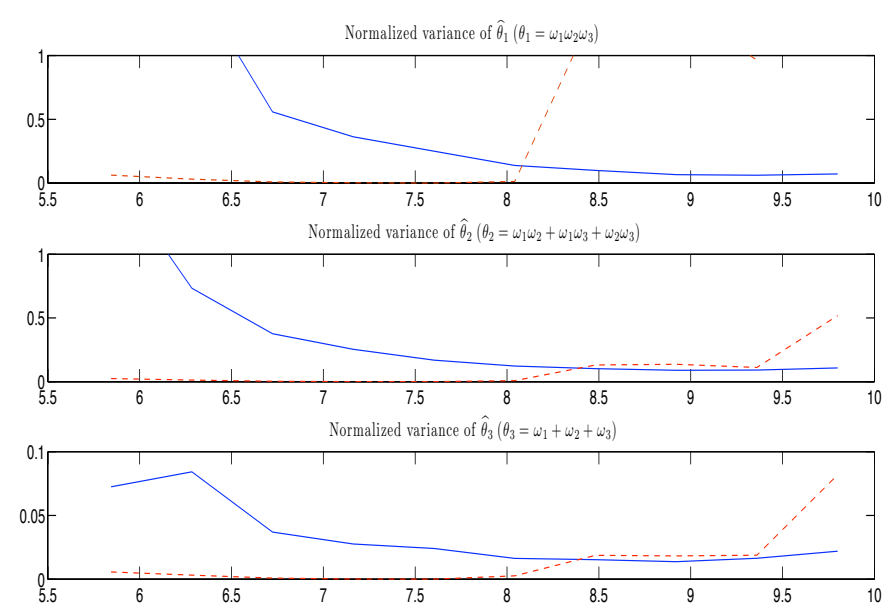

Fig. 3. Variances

[4] V. Bonnet, J. McCamley, C. Mazzà and A. Cappozzo Trunk orientation estimate during walking using gyroscope sensors, IEEE Int. Conf. on Biomedical Robotics and Biomechatronics, 2012.

[5] V. Bonnet, C. Mazzà, P. Fraisse and A. Cappozzo, A Least-Squares Identification Algorithm For Estimating Squat Exercise Mechanics Using A Single Inertial Measurement Unit, Journal of Biomechanics, 2012.

[6] A. Cappozzo, Minimum measured-input models for the assessment of motor ability, Journal of Biomechanics, vol. 35, pp. 437-446, 2002.

[7] C. Mazzà, M. Donati, J. McCamley, P. Picerno and A. Cappozzo, An optimized Kalman filter for the estimate of trunk orientation from inertial sensors data during treadmill walking, gait and posture, vol. 35, pp. 138-142, 2002.

[8] B. Dan, E. Bouillot, A. Bengoetxea, P. Noel, A. Kahn and G. Cheron, Adaptive motor strategy for squatting in spastic diplegia, European Journal of Paediatric Neurology, vol. 3, pp. 159-165, 1999.

[9] R.F. Escamilla, Knee biomechanics of the dynamic squat exercise , Medicine and Science in Sports and Exercise, vol. 10, pp. 1556-1565, 2009.

[10] M. Fliess, Analyse non standard du bruit, C.R. Acad. Sci. Paris, ser. I, 342, pp. 797-802, 2006.

[11] M. Fliess, Critique du rapport signal à bruit en communications numériques, ARIMA, vol. 9, pp. 419-429, 2008.

[12] M. Fliess, C. Join and M. Mboup, Algebraic change-point detection, Applicable Algebra Engin. Communic. Comput., vol. 21, pp. 131-143, 2010.

[13] M. Fliess, M. Mboup, H. Mounier and H. Sira-Ramírez, Questioning some paradigms of signal processing via concrete examples, in $\mathrm{H}$. Sira-Ramírez, G. Silva-Navarro (Eds.): Algebraic Methods in Flatness,
Signal Processing and State Estimation, Editorial Lagares, México, pp. $1-21,2003$

[14] M. Fliess and H. Sira-Ramírez, An algebraic framework for linear identification. ESAIM Control Optim. Calc. Variat., vol. 9, pp. 151168, 2003.

[15] M. Fliess and H. Sira-Ramírez, Closed-loop parametric identification for continuous-time linear systems via new algebraic techniques, in $\mathrm{H}$. Garnier \& L. Wang (Eds): Identification of Continuous-time Models from Sampled Data, Springer, pp. 362-391, 2008.

[16] M. Fliess, C. Join and H. Sira-Ramírez, Non-linear estimation is easy. Int. J. Modeling Identification and Control, vol. 4, no. 1, pp. 12-27, 2008.

[17] A. C. Fry, J. C. Smith, B. K. Schilling, Effect of knee position on hip and knee torques during the barbell squat, Journal of Strength and Conditioning Research, vol. 17, pp. 629-633, 2003.

[18] D. T. P. Fong and Y. Y. Chan, The Use of Wearable Inertial Motion Sensors in Human Lower Limb Biomechanics Studies: A Systematic Review , IEEE Sensors, vol. 10, pp. 1556-1565, 2009.

[19] S. Haykin, Adaptive Filter Theory ( $2^{\text {nd }}$ ed.), Englewood Cliffs, 1991.

[20] L. Hsu, R. Ortega and G. Damm, A globally convergent frequency estimator, IEEE Trans. Automat. Control, vol. 44, pp. 698-713, 1999.

[21] M. Kahn, M. Mackisack, M. Osborne and G. K. Smyth, On the consistency of Prony's method and related algorithms, J. Comput. Graph. Statist., vol. 1, pp. 329-349, 1992.

[22] R. E. Mayagoitia, A. V. Nene and P. H. Veltink, Accelerometer and rate gyroscope measurement of kinematics: an inexpensive alternative to optical motion analysis systems, Journal of Biomechanics , vol. 35, pp. 537-542, 2002.

[23] M. Mboup, C. Join and M. Fliess, A revised look at numerical differentiation with an application to nonlinear feedback control, Proc. $15^{\text {th }}$ Medit. Conf. Control Automation - MED'2007, Athens, 2007.

[24] M. Mboup, Parameter estimation for signals described by differential equations, Applicable Analysis, vol. 88, pp. 29-52, 2009.

[25] M. Mboup, C. Join and M. Fliess, Numerical differentiation with annihilators in noisy environment, Numerical Algo., vol. 50, pp. 439 467, 2009.

[26] M. Mojiri and A. R. Bakhsahi, An adaptive notch filter for frequency estimation of a periodic signal, IEEE Trans. Automat. Control, vol. 49, pp. 314-318, 2004.

[27] A. Neves, M. Mboup and M. Fliess, An Algebraic Receiver for Full Response CPM Demodulation, VI Int. Telecom. Symp. (ITS2006), Fortaleza , CE, Brazil, 2006.

[28] M. R. Osborne and G. K. Smyth, A modified Prony algorithm for exponential function fitting, SIAM Journal on Scientific Computing, vol. 16 (1), pp. 119-138, 1995.

[29] P. A. Regalia, Adaptive IIR Filtering in Signal Processing and Control, Marcel Dekker, New York, 1995.

[30] G. M. Riche de Prony, Essai expérimental et analytique : sur les lois de la dilatabilité de fluides élastiques et sur celles de la force expansive de la vapeur de l'eau et de la vapeur de l'alcool à différentes températures, Journal de l'école polytechnique, vol. 1, no. 22, pp. 24-76, 1795.

[31] C. N. Riviere, R. S. Rader and N. V. Thakor Adaptive canceling of physiological tremor for improved precision in microsurgery, IEEE Transaction Biomedical Engineering, vol. 45, pp. 839-846, 1998.

[32] R. Roy and T. Kailath, ESPRIT-estimation of signal parameters via rotational invariance techniques, IEEE Trans. Signal Process, vol. 37, pp. 984-995, 1989.

[33] J. R. Trapero, H. Sira-Ramírez and V. F. Battle, An algebraic frequency estimator for a biased and noisy sinusoidal signal, Signal Processing, vol. 87, pp. 1188-1201, 2007.

[34] J. R. Trapero, H. Sira-Ramírez and V. F. Battle, A fast on-line frequency estimator of lightly damped vibrations in flexible structures, J. Sound Vibration, vol. 307, pp. 365-378, 2007.

[35] J. R. Trapero, M. Mboup, E. Pereira-Gonzalez and V. B. Feliu, On-line frequency and damping estimation in a single-link flexible manipulator based on algebraic identification, MED, 2008.

[36] J. R. Trapero, H. Sira-Ramírez and V. F. Batlle, On the algebraic identification of the frequencies, amplitudes and phases of two sinusoidal signals from their noisy sum, Int. J. Control, vol. 81, pp. 507-518, 2008.

[37] R. Ushirobira, W. Perruquetti, M. Mboup and M. Fliess, Estimation algébrique des paramètres intrinsèques d'un signal sinusoïdal biaisé en environnement bruité, in Proc. Gretsi, 5-9 septembre, Bordeaux, France, 2011. 